\title{
Continuous blending of SPH with finite elements *
}

\author{
Sonia Fernández-Méndez ${ }^{\mathrm{a}}$, Javier Bonet ${ }^{\mathrm{b}}$ \\ and Antonio Huerta ${ }^{a}$ \\ ${ }^{a}$ Laboratori de Càlcul Numèric (LaCàN), Departament de Matemàtica Aplicada \\ III, Universitat Politècnica de Catalunya, Mòdul C2, Jordi Girona 1-3, E-08034, \\ Barcelona, Spain \\ ${ }^{\mathrm{b}}$ Civil and Computational Engineering Center $\left(C^{2} E C\right)$, University of Wales \\ Swansea, Singleton Park, Swansea, SA2 8PP, United Kingdom
}

\begin{abstract}
This paper proposes a methodology for the continuous blending of the finite element method and Smooth Particle Hydrodynamics. The coupled approximation with finite elements and particles, and the discretization of the boundary value problem with a coupled integration, are described. An integration correction is also proposed to stabilize the solution. Some numerical examples demonstrate the applicability of the method.
\end{abstract}

Key words: SPH, Finite Elements, coupling, meshless, integration correction, continuous blending

\section{Introduction}

The special issues $[1,2]$ and the present issue demonstrate the growing interest focused in meshless methods in the last decade. Meshless methods, such as the Element-Free Galerkin method (EFG) [3], the Reproducing Kernel Particle

\footnotetext{
* Sponsored by Ministerio de Ciencia y Tecnología (grants: DPI2001-2204 and REN2001-0925-C03-01) and the Generalitat de Catalunya (grant: 2001SGR00257)

Email addresses: sonia.fernandez@upc.es (Sonia Fernández-Méndez), j.bonet@swansea.ac.uk (Javier Bonet), antonio.huerta@upc.es (Antonio Huerta).

$U R L$ : www-lacan.upc.es (Antonio Huerta).
} 
Method (RKPM) [4] or the Smooth Particle Hydrodynamics method (SPH) [5$9]$, among others, are competitive for some applications compared to classical mesh-based methods.

In particular, SPH methods are widely used for fast-transient dynamic simulations, such as explosions or impact problems, because of their low computational cost and its ability to handle severe distortions [6,7]. Other meshless methods, such as EFG or RKPM, can also deal with large distortions and go beyond Finite Element (FE) computations [10], but with a higher computational cost (due to the use of Gauss quadratures or specific techniques to accurately integrate the weak form).

Nevertheless, when remeshing is not an important issue (for instance in the absence of large distortions) FE computations are preferred by practitioners for two main reasons. First it should be noted that most of the users are familiar with FE methods, and mesh-free methods are still seen as research techniques, but this, of course, will change with time. Second, FE computations in the absence of remeshing can be very efficient from a computational point of view, and thus less costly than mesh-free computations. However, these advantages disappear when intensive remeshing is required. This is the case of problems with large distortions, impact, blast, etc. In these cases SPH goes beyond FE methods. Therefore, coupling FE and ineshiess methods seems a reasonable approach to take advantage of he best properties of both methods. FE are
employed in almost everywhere and FH nodes are replaced by particles only
in the regions where the limitations of FE become apparent. An important
effort has been dedicated to the coup ing of FE and meshless methods in
the last vears. Most of the literature is devoted to the colupling of FE with

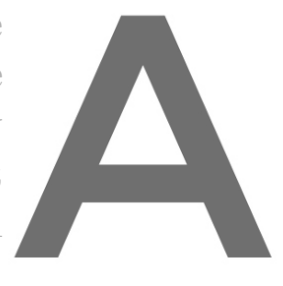

the last years. Most of the literature is devoted to the coupling of FE with watermark

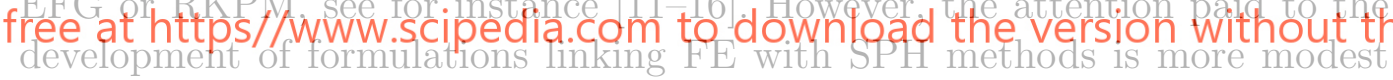
$[17,18]$.

Nowadays, there are several FE commercial or government-sponsored codes that also include SPH formulations, such as LS-Dyna, Pronto-3D, Europlexus and Sophia, among others. Nevertheless, in these codes the combination of $\mathrm{SPH}$ with FE is usually done via contact or sliding interfaces. For example, in impact problems is it usual to consider FE for the discretization of the projectile and an SPH formulation for the target, which suffers large distortions. The objective here is to discretize also the target using standard FE, and replace FE nodes by SPH particles only in regions where highly distorted elements appear during the computation. The existing contributions to link a FE region and an SPH region (with the same material) consider a transition layer of particles which are simultaneously treated as FE nodes and Lagrangian SPH particles. The influence between the different regions is taken into account with algorithmic and practical considerations. For instance, in [18] the computation of the stresses at an interface particle (node as seen from the 
FE region) is done with contributions from the interface particles and from other standard particles, but there is no contribution from the neighboring $\mathrm{FE}$ nodes. Although, as it is commented in [18], this approach may be acceptable for many applications, the mathematical basis is not clear. Consequently, the standard properties (convergence, continuity, etc), one expects form FE and $\mathrm{SPH}$ formulations, are not guaranteed with this approach.

The aim of this paper is to develop a continuous blending of SPH and FE, maintaining the reproducibility, convergence and stability properties of both methods. Several alternatives are proposed for the interpolation of a function and its derivatives, with the desired order of reproducibility (consistency) and, therefore, ensuring the convergence of the interpolation. The discretization of the problem is done with a Galerkin weak form, which is numerically integrated using Gauss quadrature in the FE region and particle integration in the rest of the domain.

Section 2 recalls some basic concepts on the SPH method. Several possibilities for the approximation of the unknown and its derivatives, coupling FE and SPH, are proposed in Section 3. In particular, Section 3.1 recalls the continuous blending method, initially proposed in [13] to blend FE and Moving Least-Squares (MLS) particle approximations. The discretization of boundary
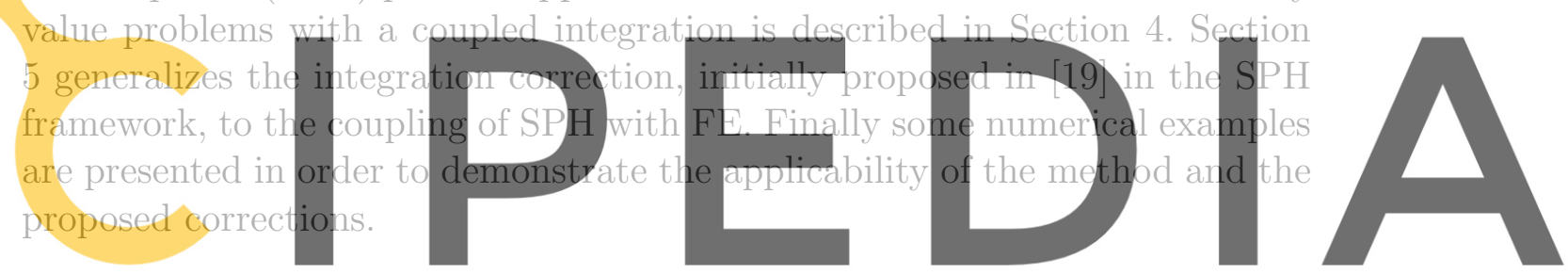

Register for free at https//www.scipedia.com to download the version without the watermark

The basis for SPH, and other particle methods, is the approximation of an arbitrary function $u(\boldsymbol{x})$ by the convolution

$$
\widehat{u}(\boldsymbol{x})=\int_{\Omega} u(\boldsymbol{s}) W(\boldsymbol{x}-\boldsymbol{s}, \rho) d \boldsymbol{s}
$$

where $W$ is a positive and compact supported function usually called kernel function or window function, see $[15,19]$. The support of $W$ is scaled by the dilation parameter $\rho$. The approximation $\widehat{u}$ will converge to the exact function as $W(\boldsymbol{x}, \rho)$ approaches the Dirac delta function $\delta(\boldsymbol{x})$. Moreover, the kernel function $W$ can be easily designed so that the approximation $\widehat{u}$ is exact for polynomials up to order $m$, that is $\widehat{u}=u$ for any $u$ polynomial of degree less or equal to $m$, see [20]. In that case, the approximation is said to have reproducibility of order $m$. Equivalently, some authors refer to this property as consistency of order $m$. 
Given a set of points in the domain $\Omega,\left\{x_{b}\right\}_{b \in \mathcal{P}}$, usually called particles, the SPH approximation is obtained from (1) with a discrete evaluation of the integral. That is, as initially proposed by Lucy [5], the SPH approximation is

$$
\widehat{u}(\boldsymbol{x})=\sum_{b \in \mathcal{P}} u\left(\boldsymbol{x}_{b}\right) W_{b}(\boldsymbol{x}) \omega_{b}
$$

where $\omega_{b}$ is a volume associated to every particle, see [5], and

$$
W_{b}(\boldsymbol{x}):=W\left(\boldsymbol{x}-\boldsymbol{x}_{b}, \rho\right)
$$

is the weight or shape function associated to particle $\boldsymbol{x}_{b}$.

Note that usually, even if approximation (1) reproduces polynomials up to order $m$, the reproducibility in the SPH approximation (2) is no longer satisfied (due to the numerical quadrature). This deficiency has important consequences for the resolution of boundary value problems in terms of accuracy, stability and convergence of the approximation.

Several possibilities have been proposed in the last decade to overcome this problem. Some of the most popular particle approximations are the Corrected Smooth Particle Hydrodynamics (CSPH) [9,19], the Renormalized Meshless Derivative [8] or the mesh-free approximations based on MLS, see [15] for a general review.
Remark 1 In the numerical resolution of a bound
SPH, the usual techniques are point collocation, or the
form with particte integration.
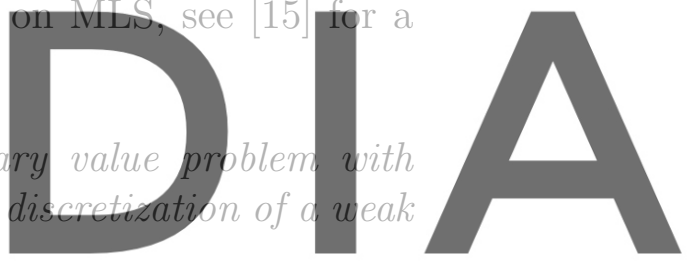

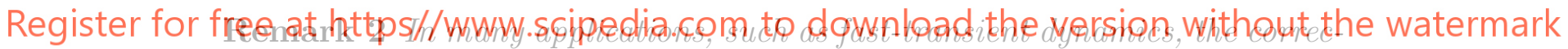
tions of the SPH method are formulated to verify reproducibility in the approximation of the derivatives, $\nabla$ u, [19]. The approximation of scalar quantities, such as the density, is done with a trivial 0-order correction.

\section{Coupled approximation}

This section is devoted to the definition of coupled approximations using finite elements and particles. As shown in Figure 1, the domain $\Omega$ is discretized with a set of particles $\left\{x_{b}\right\}_{b \in \mathcal{P}}$, marked with crosses, and a set of active nodes $\left\{x_{i}\right\}_{i \in \mathcal{N}}$, marked with black dots. Other non-active nodes are considered to define the support of the FE shape functions (thus only associated to the geometrical interpolation). In the region where only $\mathrm{FE}$ are present a standard FE approximation is used, in the region where only particles have an influence a corrected particle approximation is considered. In the transition 


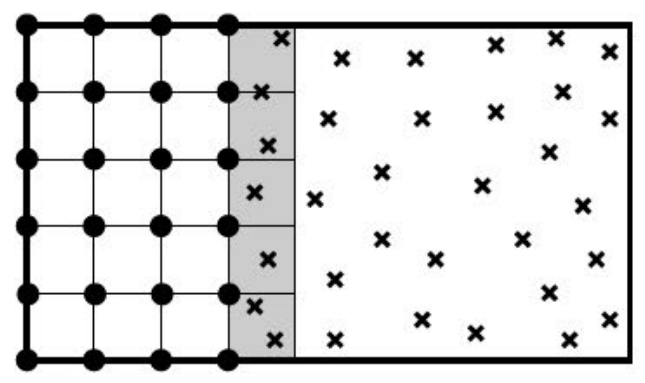

Fig. 1. FE active nodes $(\bullet)$, and transition region (in gray)

region (in gray), where both particles and nodes have an influence, a coupled interpolation is used.

In the recent years, consistent approximations coupling FE and mesh-free methods have been proposed. Seminal work on coupling FE and mesh-free methods is proposed in [11]. The continuous blending method [13] generalizes the previous ideas for any order of interpolation, suppresses the need of ramp functions, and it does not require the substitution of nodes by particles. That is, as many particles as needed can be added where they are needed, independently of the adjacent FE mesh. This is done in a hierarchical manner, see Section 3.1. Other alternatives are also possible; for instance, the bridging scale method proposed in [21] is a general technique to mix a meshfree approximation with any othe interpolation space, in particylar with FE. However, the continuous blending method presents several important advantages for the coupling of FE and mesh-fiee methods, see [16] for details. These coupled approxinations where formulated for a Galerkin formulation with accurate integration. However, these techuiques can easily be exterded to point

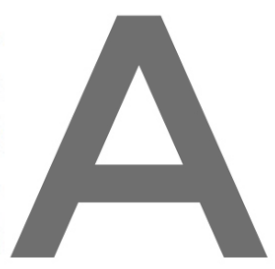
collocation or particle integration, which is the usual approach in SPH.

Register for free at https//www.scipedia.com to download the version without the watermark As commented in Remark 2, in many applications first-order reproducibility is required only for the approximation of the derivatives. Following this idea, two possibilities for the definition of coupled approximations of the derivatives are also proposed in Section 3.2.

\subsection{Continuous blending method}

In a series of papers, Huerta and coworkers $[13,22,23]$ propose a continuous blending of a MLS approximation and FE,

$$
u(\boldsymbol{x}) \simeq \widetilde{u}(\boldsymbol{x})=\sum_{i \in \mathcal{N}} N_{i}^{h}(\boldsymbol{x}) u_{i}+\sum_{b \in \mathcal{P}} \widetilde{W}_{b}(\boldsymbol{x}) u_{b}=\pi^{h} u+\sum_{b \in \mathcal{P}} \widetilde{W}_{b}(\boldsymbol{x}) u_{b}
$$

where the FE shape functions $\left\{N_{i}^{h}\right\}_{i \in \mathcal{N}}$ are as usual, and the mesh-free shape functions $\left\{\widetilde{W}_{b}\right\}_{b \in \mathcal{P}}$ take care of the reproducibility of the approximation. $\pi^{h}$ denotes the projection operator onto the finite element space. That is, the 
span of some finite element shape functions characterized by an element mesh size $h$ and associated to a set of nodes $\left\{\boldsymbol{x}_{i}\right\}_{i \in \mathcal{N}}$.

The mesh-free shape functions in (3) are defined as in standard MLS,

$$
\widetilde{W}_{b}(\boldsymbol{x})=\mathbf{P}\left(\boldsymbol{x}_{b}\right)^{T} \boldsymbol{\alpha}(\boldsymbol{x}) W_{b}(\boldsymbol{x}) \omega_{b}
$$

where $\mathbf{P}$ is a vector containing a complete polynomial base of degree less or equal to $m$. The unknown vector $\boldsymbol{\alpha}$ is determined imposing the reproducibility condition associated to the combined approximation (3), that is

$$
\mathbf{P}(\boldsymbol{x})=\pi^{h} \mathbf{P}(\boldsymbol{x})+\sum_{b \in \mathcal{P}} \widetilde{W}_{b}(\boldsymbol{x}) \mathbf{P}\left(\boldsymbol{x}_{b}\right) .
$$

This condition forces the coupled approximation (3) to exactly reproduce the polynomials in P. Substitution of (4) in (5) leads to a small system of equations for $\alpha$, see [13] for details,

$$
\mathrm{M}(x) \alpha(x)=\mathrm{P}(x)-\pi^{h} \mathrm{P}(x),
$$

\section{where}

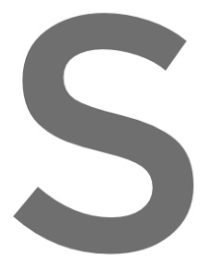

$$
\mathrm{M}(x)=\sum \mathrm{P}\left(x_{a}\right) \mathrm{P}^{T}\left(x_{a}\right) W_{a}(x) \omega_{a} .
$$
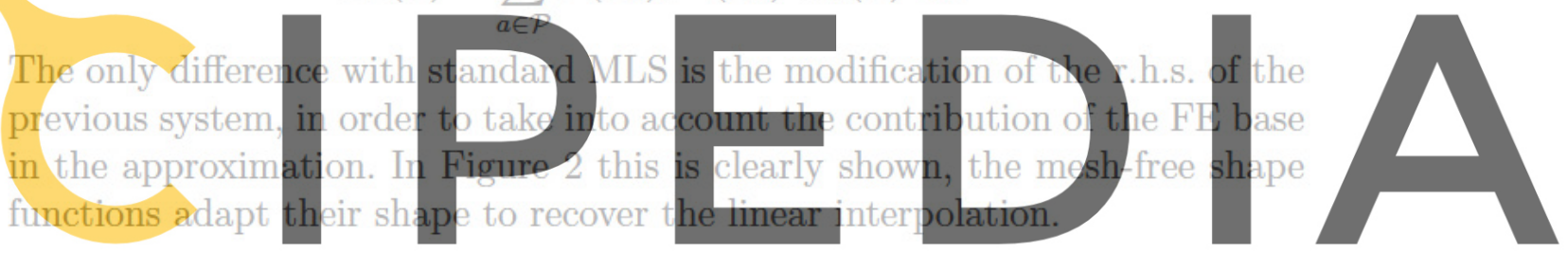

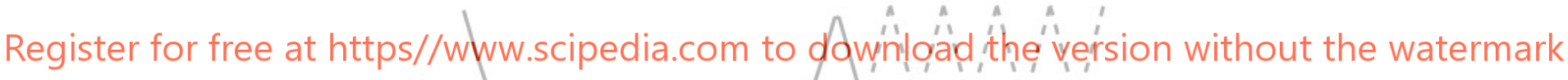

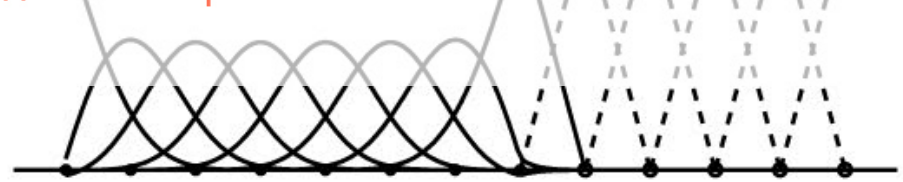

Fig. 2. Shape functions of the coupled FE (dashed line) and mesh-free (solid line) interpolation.

It is important to note that continuity of the interpolation is ensured under some conditions, even in multiple dimensions, by the following result, see [24] for demonstration.

Proposition 1 The approximation $\widetilde{u}(\boldsymbol{x})$ is continuous in $\Omega$ if:

(1) the degree of all the polynomials in $\mathbf{P}$ is less or equal to the degree of the $F E$ base (the most usual choice is $m$ equal to the degree of the FE base), and 

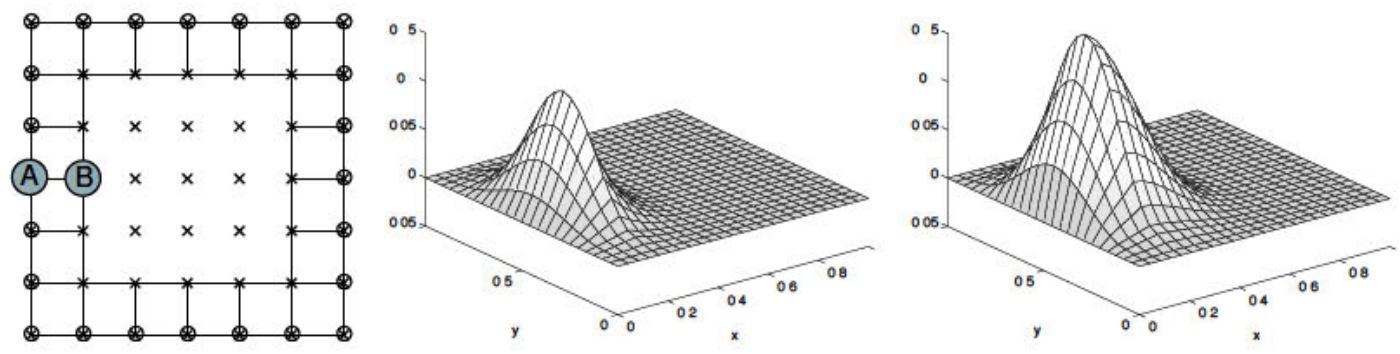

Fig. 3. Discretization with active FE nodes at the boundary (o) and particles (x), and mesh-free shape function associated to the particle located at the gray circle (A) and (B) respectively.

(2) the domain of influence of particles includes the region where FE do not have a complete interpolation basis

(i.e. , $\left\{x \in \Omega \mid \sum_{i \in \mathcal{N}} N_{i}^{h}(x) \neq 1\right\} \subseteq \bigcup_{b \in \mathcal{P}} \operatorname{supp}\left(W_{b}\right)$ ).

Note that $\sum_{i \in \mathcal{N}} N_{i}^{h}(x) \neq 1$ in the elements where at least one node is nonaetive.

Remark 3 In the regions where the FE base is complete the contribution of the particles is zero. In particular, this means that $W_{b}=0$ in the FE edges (or faces in 3D) whose nodes are all in $\mathcal{N}$ (active nodes). This is $n$ important property for the implementation of pssentid boundary conditions. If a FE mesh with active nodes at the sssential boundary free shape functions take care of reproduct at the same time, vanish at the Dirichlet boundary.

values can be imposed, as usual in the FE framework, just setting the value of

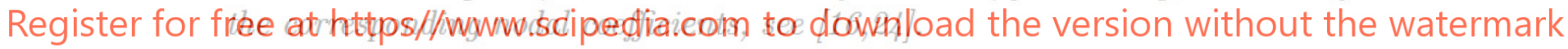

Remark 4 Obviously, the derivative of the coupled approximation (3) provides an approximation for $\boldsymbol{\nabla} u$, with reproducibility of order $m$.

In some applications first-order reproducibility is required only for the approximation of derivatives, but not for the function itself. In mechanical problems, a functional SPH approximation with 0-order reproducibility is usual for scalar quantities such as the density. In that situation, the coupled SPH-FE approximation (3) can be considered with a simple and straightforward computable expression for the SPH shape functions,

$$
\widetilde{W}_{b}(\boldsymbol{x})=W_{b}(\boldsymbol{x}) \omega_{b} \frac{\left[1-\sum_{i \in \mathcal{N}} N_{i}^{h}(\boldsymbol{x})\right]}{\sum_{a \in \mathcal{P}} W_{a}(\boldsymbol{x}) \omega_{a}} .
$$

First-order reproducibility approximations for the derivatives are presented in 
the next section.

\subsection{Coupled approximation of derivatives}

As it is commented in Remark 4, the continuous blending method provides an approximation for the derivatives with the desired reproducibility. Nevertheless, this section describes two alternative possibilities for the imposition of first-order reproducibility to the coupled approximation of $\boldsymbol{\nabla} u$. These approximations are not derived from an approximation of the function $u$ and, therefore, they are closer to the classical SPH philosophy. In both cases the situation described in Proposition 1 is assumed. That is, (1) the FE interpolation is of order greater or equal to 1 and (2) the domain of influence of particles includes the region where FE does not have a complete basis.

The first approach, similarly to [25], considers an approximation of the form

$$
\nabla u\left(\boldsymbol{x}_{a}\right) \simeq \sum_{i \in \mathcal{N}} \nabla N_{i}^{h}\left(\boldsymbol{x}_{a}\right) u_{i}+\sum_{b \in \mathcal{P}} g_{b}\left(\boldsymbol{x}_{a}\right) u_{b}
$$

where the gradient shape functions associated to the particles are defined as
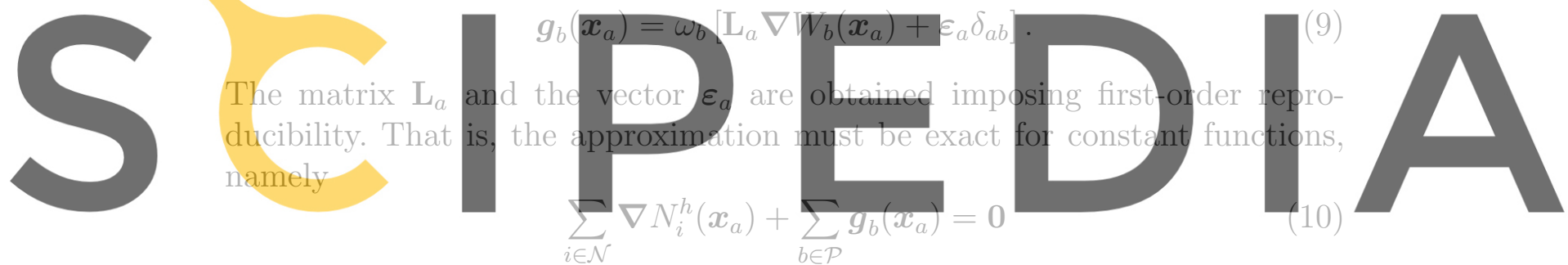

Register for free atrhitps/fwnviviscipediaj.com to download the version without the watermark

$$
\sum_{i \in \mathcal{N}} \nabla N_{i}^{h}\left(x_{a}\right) \otimes x_{i}+\sum_{b \in \mathcal{P}} g_{b}\left(x_{a}\right) \otimes x_{b}=\mathbb{I} .
$$

Thus, the expressions for $\mathbf{L}_{a}$ and $\boldsymbol{\varepsilon}_{a}$ are obtained after substitution of (9) in (10) and (11),

$$
\mathbf{L}_{a}=\left[\mathbf{I}-\sum_{i \in \mathcal{N}} \boldsymbol{\nabla} N_{i}^{h}\left(\boldsymbol{x}_{a}\right) \otimes\left(\boldsymbol{x}_{i}-\boldsymbol{x}_{a}\right)\right]\left[\sum_{b \in \mathcal{P}} \omega_{b} \boldsymbol{\nabla} W_{b}\left(\boldsymbol{x}_{a}\right) \otimes\left(\boldsymbol{x}_{b}-\boldsymbol{x}_{a}\right)\right]^{-1}
$$

and

$$
\varepsilon_{a}=\frac{1}{\omega_{a}}\left[-\sum_{i \in \mathcal{N}} \boldsymbol{\nabla} N_{i}^{h}\left(\boldsymbol{x}_{a}\right)-\mathbf{L}_{a} \sum_{b \in \mathcal{P}} \omega_{b} \boldsymbol{\nabla} W_{b}\left(\boldsymbol{x}_{a}\right)\right]
$$

The second approach, similarly to [19], considers an approximation of the form

$$
\boldsymbol{\nabla} u\left(\boldsymbol{x}_{a}\right) \simeq \sum_{i \in \mathcal{N}} \boldsymbol{\nabla} N_{i}^{h}\left(\boldsymbol{x}_{a}\right)\left[u_{i}-u_{a}\right]+\sum_{b \in \mathcal{P}} \boldsymbol{g}_{b}\left(\boldsymbol{x}_{a}\right)\left[u_{b}-u_{a}\right]
$$


where

$$
\boldsymbol{g}_{b}\left(\boldsymbol{x}_{a}\right)=\mathbf{L}_{a} \omega_{b} \nabla W_{b}\left(\boldsymbol{x}_{a}\right)
$$

Note that approximation (14) is exact for constant $u$, thus it is only necessary to impose the reproducibility condition for linear functions, that is

$$
\sum_{i \in \mathcal{N}} \boldsymbol{\nabla} N_{i}^{h}\left(\boldsymbol{x}_{a}\right) \otimes\left(\boldsymbol{x}_{i}-\boldsymbol{x}_{a}\right)+\sum_{b \in \mathcal{P}} \boldsymbol{g}_{b}\left(\boldsymbol{x}_{a}\right) \otimes\left(\boldsymbol{x}_{b}-\boldsymbol{x}_{a}\right)=\mathbf{I}
$$

After substitution of (15) in (16) the expression for $\mathbf{L}_{a}$ is obtained, which exactly coincides with (12). However, this approach does not require the computation of vector $\varepsilon_{a}$.

Remark 5 In the region where a complete FE base is present

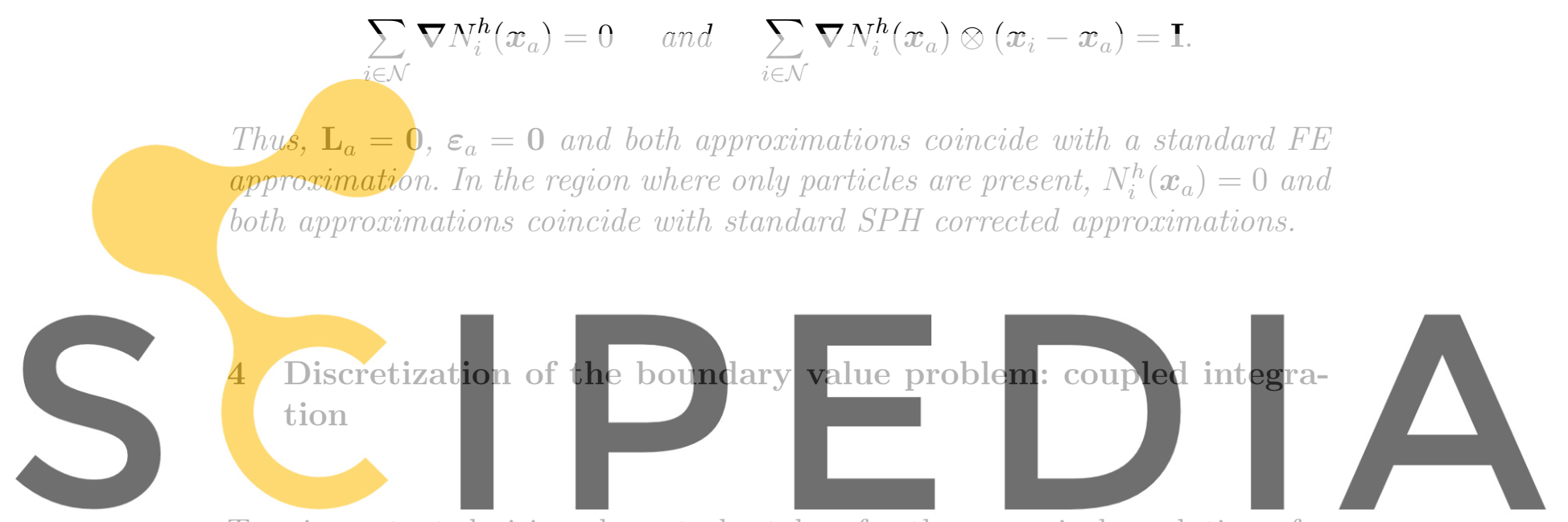

Two important decisions have to be taken for the numerical resolution of a

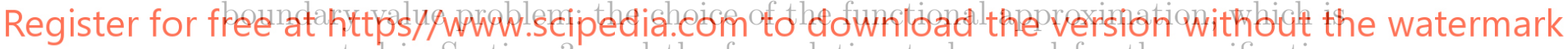
commented in Section 3, and the formulation to be used for the verification of the Partial Differential Equations (PDEs) and the imposition of boundary conditions. In SPH methods it is usual to combine a weak form of the problem with particle integration, or to consider point collocation techniques for the strong imposition of the PDEs and the boundary conditions. In both cases all the computations are done at the particles, and all the quantities of interest are stored exclusively at the particles.

This paper considers the use of a weak form with particle integration for several reasons. First of all, high order derivatives are avoided and, more important, the mathematical base of the weak form is well known, facilitating the analysis of the properties of the scheme. Furthermore, FE methods are based on the discretization of a weak form, thus, the most natural option for the coupling is considering the same weak form in the SPH region.

To show the methodology for the discretization of the problem, and for the sake of clarity, a simple model problem is considered, the Laplace equation 
with essential boundary conditions,

$$
\Delta u+f=0 \quad \text { in } \Omega, \quad u=u_{D} \quad \text { on } \partial \Omega .
$$

The above equation can be established from a variational principle defined by the potential

$$
\Pi(v)=\frac{1}{2} \int_{\Omega} \nabla v \cdot \nabla v d \Omega-\int_{\Omega} v f d \Omega .
$$

That is, the function that minimizes the potential (18) satisfies the initial Poisson problem (17).

The numerical computation of the integrals is done considering the coupled discretization of the domain $\Omega$, with FE and particles, see Figure 1 . In the FE region the standard Gauss quadrature is used in every element $\Omega_{e}$. In the rest of the domain a quadrature given by the particles, $\left\{\boldsymbol{x}_{b}\right\}_{b \in \mathcal{P}}$, and the corresponding volumes, denoted by $V_{b}$, is considered. Thus, the discrete version of the potential (18) is

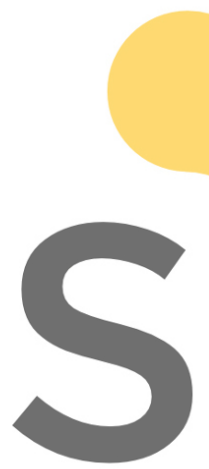

$\Pi(v)=\frac{1}{2} \sum_{e} \int_{\Omega_{e}} \nabla v \cdot \nabla v d \Omega+\frac{1}{2} \sum_{b \in \mathcal{P}} \nabla v\left(\boldsymbol{x}_{b}\right) \cdot \nabla v\left(\boldsymbol{x}_{b}\right) V_{b}$

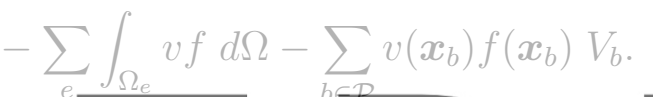

(19)
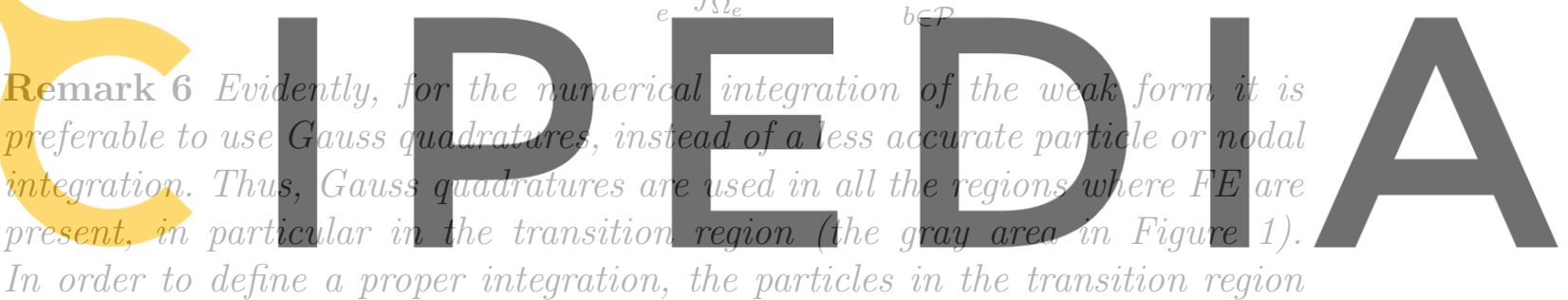

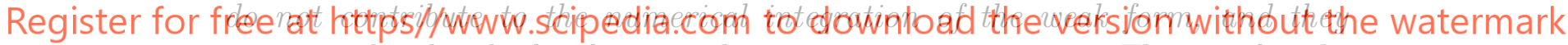
are considered only for functional approximation purposes. That is, for the particles in the transition region, the integration weight is zero, $V_{b}=0$, but they do contribute in the computation of the functional approximation, $\omega_{b} \neq 0$.

Remark 7 Essential boundary conditions can be easily enforced if a coupled discretization with FE nodes is used along the boundary. In that case, the prescribed values can be imposed in a standard manner, as in the FE framework, just setting the value of the corresponding nodal coefficients. Recall that meshfree approximations do not verify the delta Kronecker property and, therefore, the enforcement of prescribed values can not be performed directly. The alternative is to use a modified variational principle in order to take into account the essential boundary condition, such as the penalty method, the method of Lagrange multipliers or Nitsche's method, see [24] for a general presentation.

The discretization of the boundary value problem is obtained after substitution of the functional approximations in the discrete version of the potential (19). In order to simplify the notation, let us consider approximations that can be 
written in the form

$$
v(\boldsymbol{x}) \simeq \widetilde{v}(\boldsymbol{x})=\sum_{k \in \mathcal{N} \cup \mathcal{P}} v_{k} N_{k}(\boldsymbol{x}), \quad \boldsymbol{\nabla} v(\boldsymbol{x}) \simeq \boldsymbol{\nabla} \widetilde{v}(\boldsymbol{x})=\sum_{k \in \mathcal{N} \cup \mathcal{P}} v_{k} \boldsymbol{g}_{k}(\boldsymbol{x})
$$

where the FE shape functions are as usual, $N_{k}:=N_{k}^{h}$ and $\boldsymbol{g}_{k}:=\nabla N_{k}^{h}$ for $k \in \mathcal{N}$. The SPH shape functions are $N_{k}:=\widetilde{W}_{k}$ for $k \in \mathcal{P}$, as defined in Section 3.1 by (4), or by (7) for 0-order reproducibility. The gradient SPH shape functions can be defined by $\boldsymbol{g}_{k}=\nabla \widetilde{W}_{k}$ or by equations (9), (12) and (13). The consideration of other approximation expressions, such as (14), does not imply any added difficulty.

Substitution of (20) in (19) leads to the discrete version of the potential,

$$
\Pi(\mathbf{v})=\frac{1}{2} \mathbf{v}^{T} \mathbf{K} \mathbf{v}-\mathbf{f}^{T} \mathbf{v},
$$

where

$$
K_{i j}=\sum_{e} \int_{\Omega_{e}} \boldsymbol{g}_{i} \cdot \boldsymbol{g}_{j} d \Omega+\sum_{b \in \mathcal{P}} \boldsymbol{g}_{i}\left(\boldsymbol{x}_{b}\right) \cdot \boldsymbol{g}_{j}\left(\boldsymbol{x}_{b}\right) V_{b}, \quad \text { for } i, j \in \mathcal{N} \cup \mathcal{P}
$$

and

$$
f_{i}=\sum_{e} \int_{\Omega_{e}} N_{i} f d \Omega+\sum_{b \in \mathcal{P}} N_{i}\left(\boldsymbol{x}_{b}\right) f\left(\boldsymbol{x}_{b}\right) V_{b}, \quad \text { for } i \in \mathcal{N} \cup \mathcal{P} .
$$

The system of equations is obtained after minimization with respect to the coefficients $\mathbf{v}$, subject to the restrictions due to the essential boundary conditions [24].

\section{Integration correction}

As usual in SPH methods, the solution of boundary value problems coupling FE and SPH can suffer from instabilities that drastically degrade the results. There are spurious modes present in the solution as a consequence of the inaccurate particle integration. Bonet and coworkers pointed out in [19] the importance of ensuring that meshless methods pass the patch test. They also proposed a necessary condition to be satisfied by the discretization of the integrals and functional approximations. This condition corresponds to the discrete integrated version of

$$
\int_{\Omega} \boldsymbol{\nabla} N_{a} d \Omega=\int_{\partial \Omega} \boldsymbol{n} N_{a} d \Gamma, \quad \text { for } a \in \mathcal{N} \cup \mathcal{P}
$$

where $\boldsymbol{n}$ is the unitary outward normal vector. 
Note that, although (21) is satisfied due to the so-called Green or gradient theorem, the fulfillment of its discrete integrated SPH version is not guaranteed due to the particle integration errors.

In the context of the coupled method proposed in Section 3, the discrete integrated version of (21) must be satisfied for all the shape functions, corresponding to both nodes and particles.

For a FE shape function, $a \in \mathcal{N}$, condition (21) corresponds to

$$
\sum_{e} \int_{\Omega_{e}} \nabla N_{a}^{h} d \Omega=\sum_{E} \int_{\Gamma_{E}} \boldsymbol{n} N_{a}^{h} d \Gamma
$$

where $\Omega_{e}$ and $\Gamma_{E}$ are $\mathrm{FE}$ in $\Omega$ and $\partial \Omega$ respectively, and the integration is approximated with Gauss quadrature in every element. Note that the summations of integrals in $\Omega_{e}$ and $\Gamma_{E}$ in equation (22) are exactly equal to the integrals in $\Omega$ and $\partial \Omega$ in equation (21). Thus, (22) is automatically satisfied for all FE nodes, $a \in \mathcal{N}$.

This is not the case for a particle shape function. Due to the errors of the particle integration the discrete version of (21) is not satisfied, that is, in general, for $a \in \mathcal{P}$

$$
\begin{aligned}
\sum_{e} \int_{\Omega_{e}} \boldsymbol{g}_{a} d \Omega+\sum_{b \in \mathcal{P}} \boldsymbol{g}_{a}\left(\boldsymbol{x}_{b}\right) V_{b} & \\
& \neq \sum_{E} \int_{\Gamma_{E}} \boldsymbol{n} \widetilde{W}_{a} d \Gamma+\sum_{B \in \mathcal{P}^{B}} \boldsymbol{n}_{B} \widetilde{W}_{a}\left(\boldsymbol{x}_{B}\right) A_{B}
\end{aligned}
$$

where $\mathcal{P}^{B}$ denotes the indexes of the particles in the region of the boundary $\partial \Omega$ where no FE are present, and $A_{B}$ is the tributary area of particle $\boldsymbol{x}_{B}$.

To overcome this problem, and following the idea proposed in [19], the computation of the gradient at every particle is modified by the introduction of an integration correction vector $\gamma=\left\{\gamma_{b}\right\}_{b \in \mathcal{P}}$,

$$
\widetilde{\nabla} u\left(\boldsymbol{x}_{b}\right)=\boldsymbol{\nabla} \widetilde{u}\left(\boldsymbol{x}_{b}\right)+\gamma_{b} \llbracket u \rrbracket_{b} \quad \text { for } b \in \mathcal{P},
$$

where

$$
\llbracket u \rrbracket_{b}=u_{b}-\sum_{a} u_{a} \widetilde{W}_{a}\left(\boldsymbol{x}_{b}\right)
$$

Remark 8 Note that, assuming m-order reproducibility for the interpolation, the bracket $\llbracket \cdot \rrbracket_{b}$ vanishes for all polynomials of degree less or equal to $m$ and, therefore, for these polynomials the evaluation of the gradient will not be affected by the correction. That is, the corrected approximation of the gradient maintains the m-order reproducibility for any $\gamma_{b}$. 
The corrected approximation of the gradient at any particle can also be written as

$$
\widetilde{\nabla} u\left(\boldsymbol{x}_{b}\right)=\sum_{a} u_{a} \widetilde{\boldsymbol{g}}_{a}\left(\boldsymbol{x}_{b}\right),
$$

with the definition of the corrected shape functions

$$
\widetilde{\boldsymbol{g}}_{a}\left(\boldsymbol{x}_{b}\right)=\boldsymbol{g}_{a}\left(\boldsymbol{x}_{b}\right)-\gamma_{b} \widetilde{W}_{a}\left(\boldsymbol{x}_{b}\right)+\gamma_{b} \delta_{a b}
$$

The integration correction vectors $\gamma_{b}$ are determined imposing that the equality in (23) is fulfilled, that is

$$
\begin{aligned}
\sum_{e} \int_{\Omega_{e}} \boldsymbol{g}_{a} d \Omega+ & \sum_{b \in \mathcal{P}} \widetilde{\boldsymbol{g}}_{a}\left(\boldsymbol{x}_{b}\right) V_{b} \\
& =\sum_{E} \int_{\Gamma_{E}} \boldsymbol{n} \widetilde{W}_{a} d \Gamma+\sum_{B \in \mathcal{P}^{B}} \boldsymbol{n} \widetilde{W}_{a}\left(\boldsymbol{x}_{B}\right) A_{B} \quad \text { for } a \in \mathcal{P} .
\end{aligned}
$$

After substitution of (24) in (25) the linear system of equations to be solved is obtained,

$$
\begin{aligned}
\sum_{b \in \mathcal{P}} V_{b}\left[\delta_{a b}-\widetilde{W}_{a}\left(\boldsymbol{x}_{b}\right)\right] \boldsymbol{\gamma}_{b}= & -\sum_{b \in \mathcal{P}} V_{b} \boldsymbol{g}_{a}\left(\boldsymbol{x}_{b}\right)-\sum_{e} \int_{\Omega_{e}} \boldsymbol{g}_{a} d \Omega \\
& +\sum_{B \in \mathcal{P}^{B}} A_{B} \boldsymbol{n}_{B} \widetilde{W}_{a}\left(\boldsymbol{x}_{B}\right)+\sum_{E} \int_{\Gamma_{E}} \boldsymbol{n} \widetilde{W}_{a} d \Gamma \quad \text { for } a \in \mathcal{P} .
\end{aligned}
$$

Thus, the computation of the integration correction vectors requires the resolution of a linear system of equations for each spatial dimension, but with the same matrix

$$
A_{a b}=V_{b}\left[\delta_{a b}-\widetilde{W}_{a}\left(\boldsymbol{x}_{b}\right)\right] \quad \text { for } a, b \in \mathcal{P} .
$$

Remark 9 The behavior of the correction coefficients $\gamma_{b}$ (order of magnitude, sensitivity to small perturbations of the particle distribution, etc) is the same as in standard SPH methods. This is the subject of current study and a possible future publication [26].

\section{$6 \quad$ Numerical examples}

Two numerical examples are presented in this section to demonstrate the applicability and performance of the proposed methodology. First an academic Poisson problem with known analytical solution is considered. Numerical instabilities are present in the solution due to particle integration, and the integration correction technique presented in Section 5 is used to improve the results. On the other hand, a fast-transient dynamics simulation with severe distortions is considered for the second numerical example. The replacement 
of highly distorted FE by particles allows going beyond standard FE computations. In both examples the use of a layer of $\mathrm{FE}$ nodes along the boundary facilitates the implementation of essential boundary conditions.

\subsection{Poisson problem}

The Laplace equation with Dirichlet boundary conditions is considered next,

$$
\left\{\begin{array}{cl}
-\Delta u=f & \text { in } \Omega=[0,1] \times[0,1] \\
u=0 & \text { on } \partial \Omega
\end{array}\right.
$$

with source term $f=2[y(1-y)+x(1-x)]$.
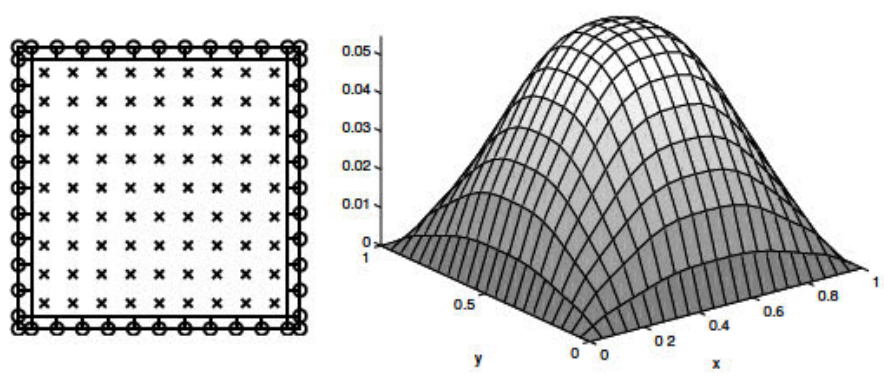

Fig. 4. Discretization with nodes (o) and a regular distribution of particles $(\times)$, and solution coupling FE and SPH

Figure 4 shows a discretization coupling a layer of $\mathrm{FE}$ along the boundary with a regular distribution of particles. The problem is solved using the continuous blending method described in Section 3.1 with the discretization of the boundary value problem commented in section 4 . The nodes along the boundary allow to impose the prescribed values, as usual in the FE framework, just setting the corresponding nodal coefficient. Apart from that, in this example the performance of the coupled approximation is the same as in a standard SPH method. The coupling is done in a continuous way and preserves the properties of FE and SPH methods. Accurate and stable results are obtained when a regular distribution of particles is used.

However, the typical instabilities of the SPH method appear when a nonregular distribution of particles is used. In figure 5 the same pattern of particles and $\mathrm{FE}$ is considered, but with a small perturbation for the position of the particles. As usual in SPH methods, due to particle integration, the solution with a random distribution of particles presents instabilities, which drastically degrade the accuracy. The solution is improved with the integration correction, 

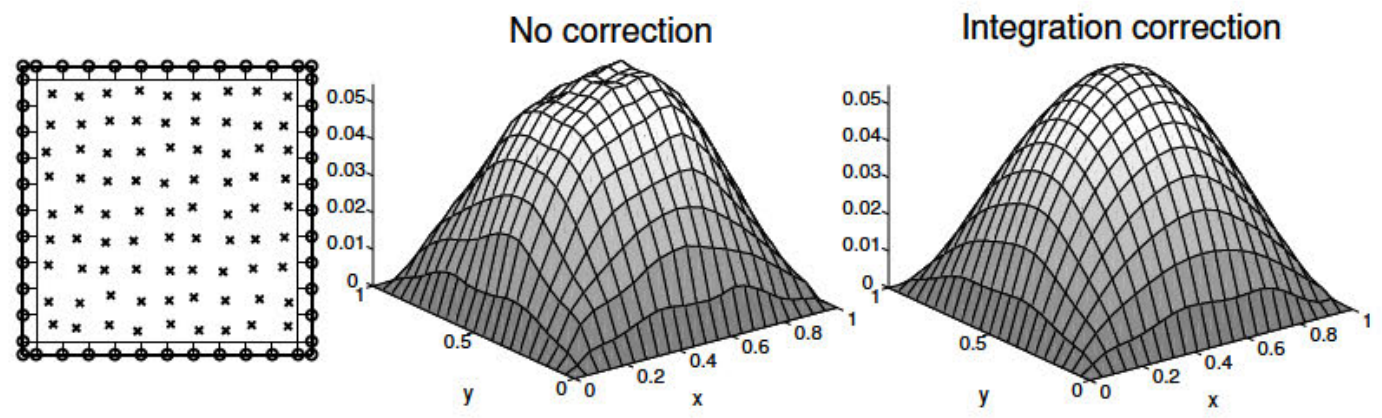

Fig. 5. Discretization with nodes $(\mathrm{o})$ and particles $(\times)$, solution without stabilization (center) and stabilized solution with integration correction (right).

initially proposed in [19] for SPH methods and extended for the coupling of $\mathrm{FE}$ and SPH in Section 5.

\subsection{Fast-transient dynamics example}

The rigid punch test described in Figure 6 is considered next. A plane strain quasi-incompressible Neo-Hookean material model is used with density $\rho=$ $10^{3} \mathrm{Kg} / \mathrm{m}^{3}$, bulk modulus $k=1.667 \cdot 10^{6} \mathrm{~N} / \mathrm{m}^{2}$ and shear modulus $\mu=$ $0.3571 \cdot 10^{6} \mathrm{~N} / \mathrm{m}^{2}[27]$. Given the symmetries, only a quarter of the domain is

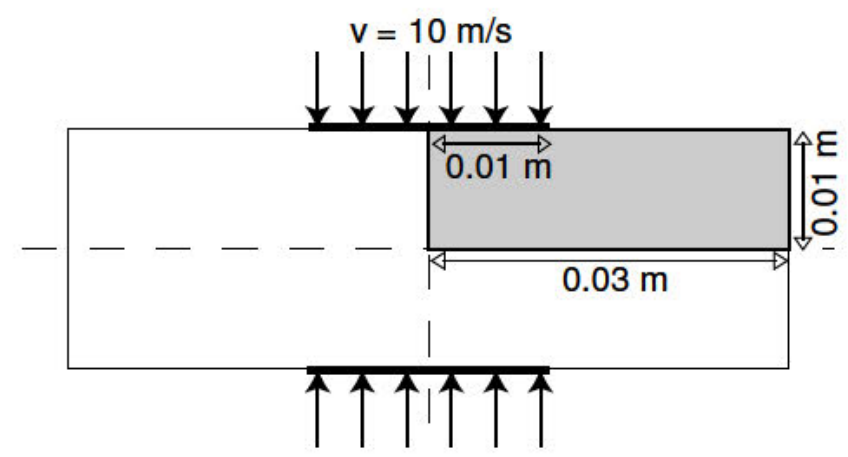

Fig. 6. Rigid punch problem statement

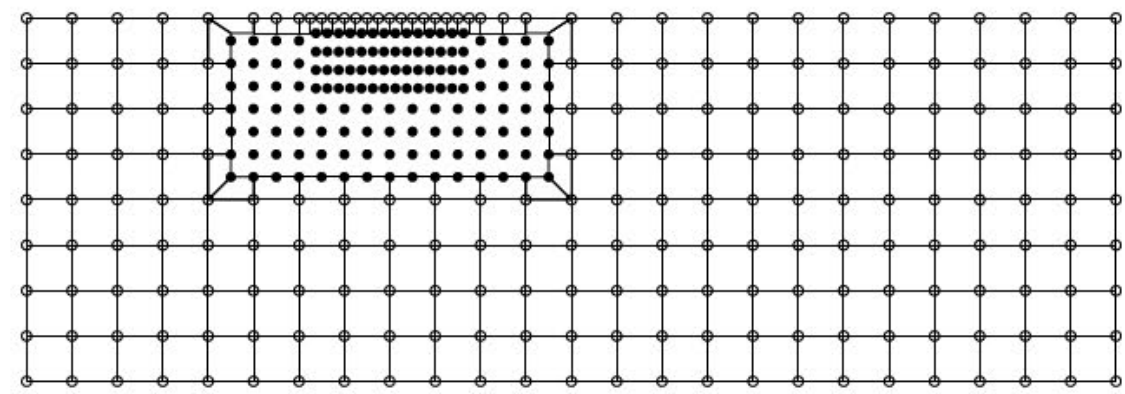

Fig. 7. Discretization of a quarter of the domain coupling FE and SPH. Particles are marked with $\bullet$ and active nodes are marked with 0. 
discretized. The material is stretched to the $60 \%$ of its original length.
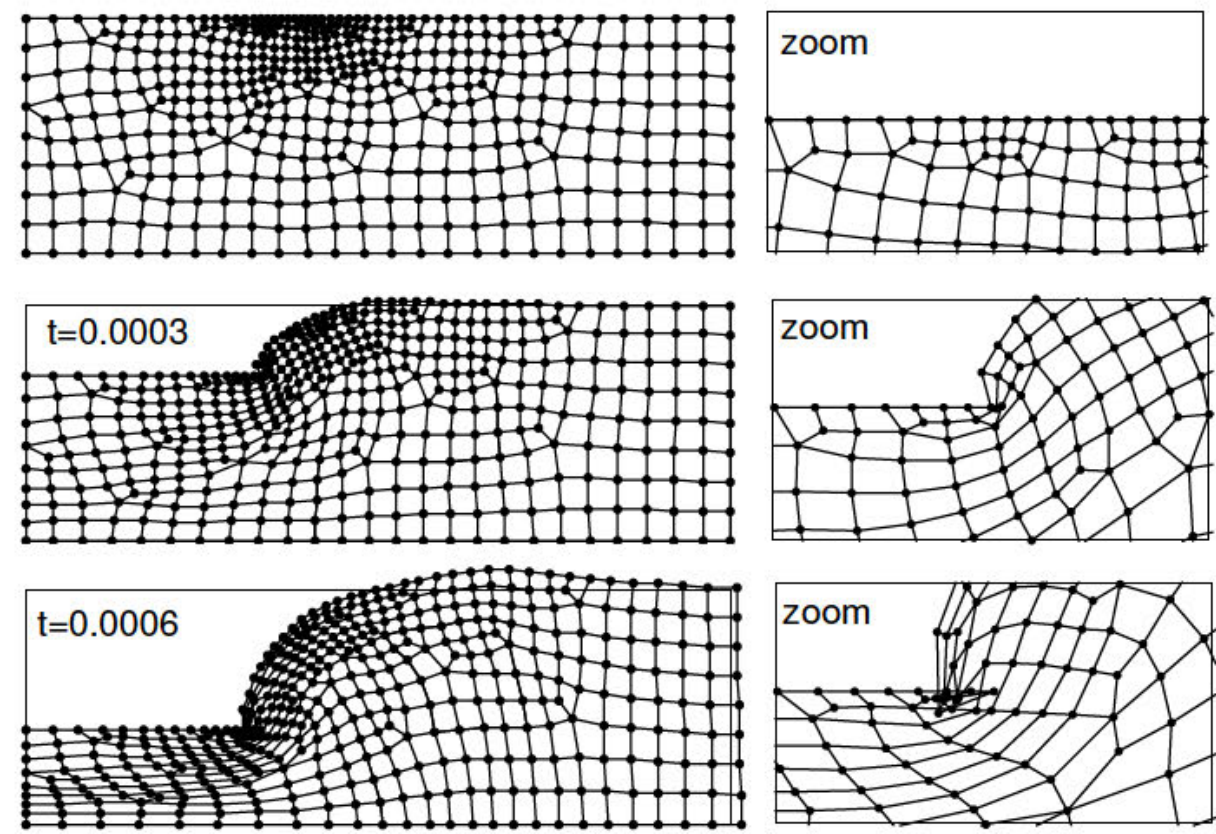

Fig. 8. Solution with linear FE at $t=0, t=0.0003$ and $t=0.0006$, and zoom centered on the material point initially located at $(0.01,0.01)$.
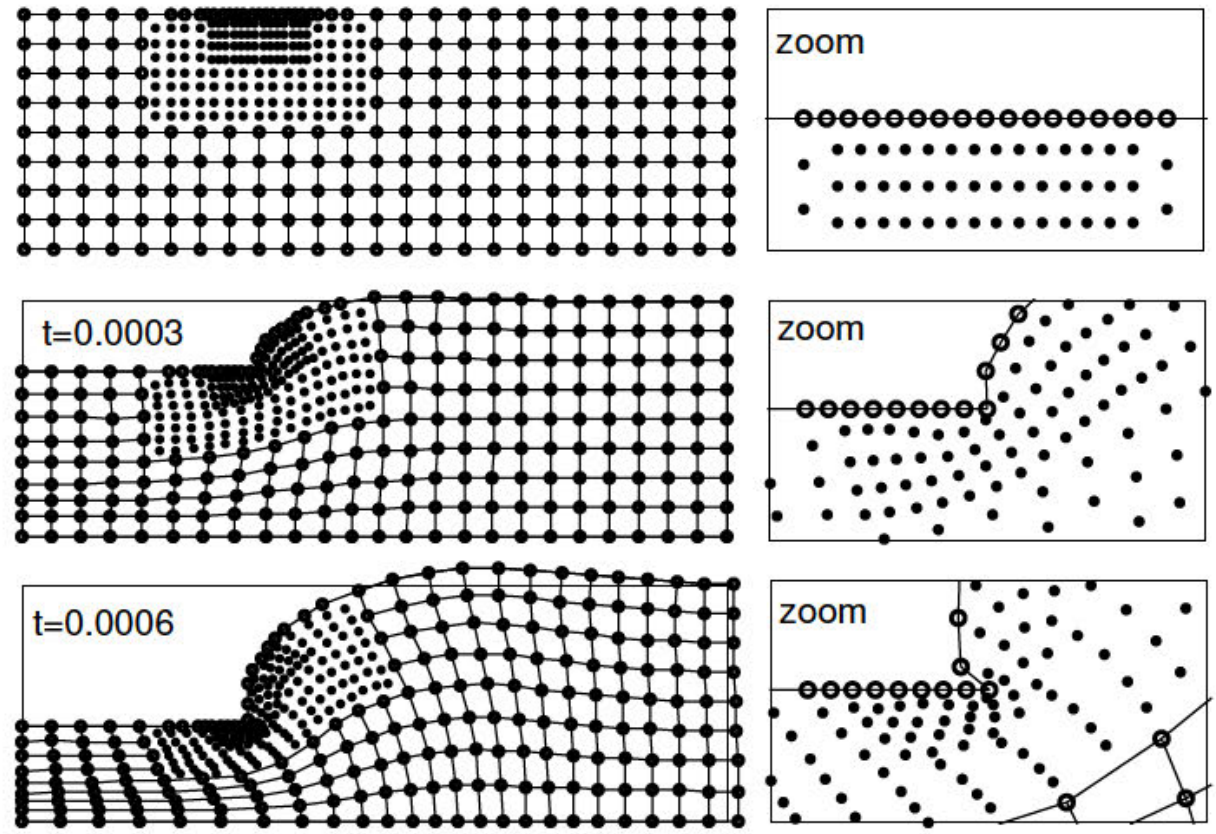

Fig. 9. Solution with FE and SPH, and zoom centered on the material point initially located at $(0.01,0.01)$.

Figure 8 shows the solution obtained with a linear FE discretization. The mesh has been refined near the punch region in orden to obtain an accurate 
solution. However, the solution is degraded due to the presence of too distorted elements in the punch region, see the zooms on the right.

Figure 9 shows the solution obtained using the coupling of SPH and FE. The continuous blending method is used for the approximation of the displacements and its derivatives. Figure 7 shows the discretization with particles and FE, with active and non-active nodes. As usual in mesh-free methods [10], the use of an SPH approximation near the punch region allows to go beyond the FE computations. The SPH discretization avoids the problems due to the distortion of the FE.

It is important to note that with an SPH discretization the refinement of the discretization near the punch region is easily performed, with no remeshing cost.

Remark 10 Although it is not exploited in this example, the definition of the neighborhood between particles in the SPH region can be updated during the computations, leading to an updated Lagrangian formulation. The update of the "connectivity" between particles does not imply the usual projection errors of the FE computations (due to the projection of quantities between meshes). This is an important issue in problems with severe distortions, brittle fracture, etc.

Remark 11 Obviously, the continuous blending of SPH and FE inherits the stability deficiencies of the SPH method. In problems with extremely large distortions, the SPH Lagrangian formulation will need updates of the reference configuration. Then, zero energy modes are more likely to be excited. When a reduced number of updates are necessary the error is small and can go unnoticed, but when frequent updates are performed the solution can be completely spoilt due to zero energy modes in the SPH approximation. To avoid numerical fracture in high-tension zones, standard techniques can be implemented [28-30]. Other stabilization techniques are currently under investigation and will be discussed in a forthcoming publication [31].

On the other hand, as it is seen in the example, a layer of FE can be used along the punch boundary in order to facilitate the enforcement of essential boundary conditions, or contact boundary conditions in a more general case.

\section{Conclusions}

The examples demonstrate the applicability of the proposed methodology for the coupling of SPH and FE. 
Apart from the obvious advantages of SPH for adaptivity processes, the use of an SPH discretization in the regions where severe distortions are present allows to go beyond FE computations. If needed, the "connectivity" between particles in the SPH region can be updated during the computations, without projection errors. In this situation, the continuous blending of $\mathrm{SPH}$ and FE inherits the stability properties of the SPH considered formulation.

On the other hand, the use of a layer of FE along the boundary, coupled with the SPH interpolation, facilitates the enforcement of essential boundary conditions, or contact boundary conditions.

The coupling of FE and mesh-free methods maintains the low computational cost of both methods, and takes advantage of their best properties. This method seems to be very promising for the solution of fast-transient dynamics problems, impact simulations, brittle fracture or explosions.

Finally, the integration correction avoids, or at least attenuates, the presence of the possible instabilities in the SPH numerical solution (due to the particle integration).

\section{References}

[1] W. K. Liu, T. Belytschko, J. T. Oden, editors, Meshless methods, Comput. Methods Appl. Mech. Eng. 139 (1-4) (1996) 1-440.

[2] J. S. Chen, W. Liu, editors, Meshless methods: Recent advances and new applications, Comput. Methods Appl. Mech. Eng. 193 (12-14) (2004) 933-1321.

[3] T. Belytschko, Y. Y. Lu, L. Gu, Element free galerkin methods, Int. J. Numer. Methods Eng. 37 (2) (1994) 229-256.

[4] W. K. Liu, S. Jun, S. Li, J. Adee, T. Belytschko, Reproducing kernel particle methods for structural dynamics, Int. J. Numer. Methods Eng. 38 (10) (1995) $1655-1679$.

[5] L. Lucy, A numerical approach to the testing of the fission hypothesis, Astron. J. 82 (1977) 1013-1024.

[6] J. W. Swengle, D. L. Hicks, S. W. Attaway, Smoothed particle hydrodynamics stability analysis, J. Comput. Phys. 116 (1995) 123-134.

[7] G. R. Johnson, S. R. Beissel, Normalized smoothing functions for SPH impact computations, Comput. Methods Appl. Mech. Eng. 39 (16) (1996) 2725-2741.

[8] J. P. Vila, On particle weighted methods and smooth particle hydrodynamics, Math. Models Methods Appl. Sci. 9 (2) (1999) 161-209. 
[9] J. Bonet, T. Lok, Variational and momentum preservation aspects of smooth particle hydrodynamic formulations, Comput. Methods Appl. Mech. Eng. 180 (1-2) (1999) 97-115.

[10] S. Jun, W. K. Liu, T. Belytschko, Explicit reproducing kernel particle methods for large deformation problems, Int. J. Numer. Methods Eng. 41 (1998) 137-166.

[11] T. Belytschko, D. Organ, Y. Krongauz, A coupled finite element-element-free Galerkin method, Comput. Mech. 17 (3) (1995) 186-195.

[12] D. Hegen, Element free galerkin methods in combination with finite element approaches, Comput. Methods Appl. Mech. Eng. 135 (1-2) (1996) 143-166.

[13] A. Huerta, S. Fernández-Méndez, Enrichment and coupling of the finite element and meshless methods, Int. J. Numer. Methods Eng. 48 (11) (2000) 1615-1636.

[14] G. J. Wagner, W. K. Liu, Hierarchical enrichment for bridging scales and meshfree boundary conditions, Int. J. Numer. Methods Eng. 50 (3) (2001) 507-524.

[15] T. Belytschko, A. Huerta, S. Fernández-Méndez, T. Rabczuk, Encyclopedia of Computational Mechanics, Vol. 1, John Wiley \& Sons, 2004, Ch. 10 (Meshless methods).

[16] A. Huerta, S. Fernández-Méndez, W. K. Liu, A comparison of two formulations to blend finite elements and mesh-free methods, Comput. Methods Appl. Mech. Eng. 193 (12-14) (2004) 1105-1117.

[17] S. Attaway, M. Heinstein, J. Swegle, Coupling of smoothed particle hydrodynamics with the finite element method, Nuclear Eng. Design 150 (1994) 199-205.

[18] G. R. Johnson, R. A. Stryk, S. R. Beissel, SPH for high velocity impact computations, Comput. Methods Appl. Mech. Eng. 39 (16) (1996) 2725-2741.

[19] J. Bonet, S. Kulasegaram, Correction and stabilization of smooth particle hydrodynamics methods with applications in metal forming simulations, Int. J. Numer. Methods Eng. 47 (6) (2000) 1189-1214.

[20] W. K. Liu, Y. Chen, S. Jun, J. S. Chen, T. Belytschko, C. Pan, R. A. Uras, C. T. Chang, Overview and applications of the reproducing kernel particle methods, Arch. Comput. Methods Engrg. 3 (1) (1996) 3-80.

[21] G. J. Wagner, W. K. Liu, Application of essential boundary conditions in meshfree methods: a corrected collocation method, Int. J. Numer. Methods Eng. 47 (8) (2000) 1367-1379.

[22] A. Huerta, S. Fernández-Méndez, P. Díez, Enrichissement des interpolations d' éléments finis en utilisant des méthodes de particules, ESAIM-Math. Model. Numer. Anal. 36 (6) (2002) 1027-1042.

[23] S. Fernández-Méndez, P. Díez, A. Huerta, Convergence of finite elements enriched with meshless methods, Numer. Math. 96 (1) (2003) 43-59. 
[24] S. Fernández-Méndez, A. Huerta, Imposing essential boundary conditions in mesh-free methods, Comput. Methods Appl. Mech. Eng. 193 (12-14) (2004) $1257-1275$.

[25] J. Bonet, S. Kulasegaram, M. Rodríguez-Paz, Variational formulation for the smooth particle hydrodynamics (SPH) simulation of fluid and solid problems, Comput. Methods Appl. Mech. Eng. 193 (12-14) (2004) 1245-1256.

[26] S. Fernández-Méndez, J. Bonet, On the regularity and convergence of the integration correction in SPH. In preparation.

[27] J. Bonet, R. D. Wood, Nonlinear continuum mechanics for finite element analysis, Cambridge University Press, Cambridge, 1997.

[28] P. Randles, L. Libersky, Normalized SPH with stress points., Int. J. Numer. Methods Eng. 48 (10) (2000) 1445-1462.

[29] P. W. Randles, A. G. Petschek, L. D. Libersky, C. T. Dyka, Stability of DPD and SPH., in: M. Griebel, M. A. Schweitzer (Eds.), Meshfree methods for partial differential equations, Vol. 26 of Lecture Notes in Computational Science and Engineering, Springer-Verlag, Berlin, 2002, pp. 117-129, papers from the International workshop, Universität Bonn, Germany, September 11-14, 2001.

[30] T. Belytschko, S. Xiao, Stability analysis of particle methods with corrected derivatives., Comput. Math. Appl. 43 (3-5) (2002) 329-350.

[31] Y. Vidal, Mesh-free methods for dynamic problems: incompressibility and large strains, Ph.D. thesis, Universitat Politècnica de Catalunya, Barcelona, Spain (2005). 